\title{
FORCE AND DISPLACEMENT INFLUENCE FUNCTIONS FOR THE CIRCULAR RING
}

\author{
J. R. BARBER Department of Mechanical Engineering, University of Newcastle upon Tyne. Member of \\ the Institution
}

\begin{abstract}
A set of influence functions is developed for the complete, uniform, circular ring, subjected to concentrated forces or moments. Equilibrium is satisfied by the introduction of fictitious supports that are self-cancelling when the applied force system is in equilibrium. The method provides a general solution for the circular ring on a statically determinate support.
\end{abstract}

\section{INTRODUCTION}

If a complete circular ring is subjected to a known, selfequilibrating system of forces, the equations of equilibrium are not sufficient to determine the internal forces and moments. In certain cases, the defect can be made up by arguments from symmetry, but it is usually necessary to impose the condition of continuity of displacements around the ring. This condition is often greatly simplified by the use of Castigliano's theorem, but the solution can be tedious in asymmetric practical problems, particularly if a number of concentrated forces and moments are applied to the ring.

In the design field, it is usual to build up the solution to such a problem by superposition. Lists of elementary solutions appropriate to the complete circular ring are given by Roark (1) $\dagger$ (in-plane loading only) and Blake (2) (in-plane and transverse loading). However, neither of these authors gives a set of influence functions that is sufficiently general to cover all possible problems. In this paper, a set of functions is given that satisfies this requirement of generality.

It is assumed throughout that the cross-section of the ring is uniform and small in both directions in comparison with the mean diameter. It is also assumed that extension of the centre-line and shear deflection can be neglected.

\section{THE FICTITIOUS SUPPORT SYSTEM}

The simplest system of influence functions would be that corresponding to the application of a force in an arbitrary direction and a moment about an arbitrary axis to a given point on the ring. However, this system of forces is not self-equilibrating, and if we select any particular system of supports to oppose the load (as is done by Roark (1) and Blake (2)) we immediately lose the generality of the method.

This difficulty can be overcome by introducing 'fictitious supports' and applying the real support reactions in a particular problem as additional external loads. The fictitious supports can be so chosen that their total effect on the system is self-cancelling whenever the applied external forces are in equilibrium. For the results given

\footnotetext{
The MS. of this paper was received at the Institution on 27th October

1976 and accepted for publication on 13th September 1977.

$\dagger$ References are given in Appendix 2.
}

here, forces acting through the ring centre are supported by equal and opposite reactions uniformly distributed around the ring; moments about the ring axis by a uniform tangential load; and transverse moments by a sinusoidally-varying distributed transverse load. This system of fictitious support reactions is illustrated in Fig. 1.

This choice of fictitious support is identical with the system of 'inertia forces' which would be developed if the ring were actually allowed to accelerate as a rigid body under the influence of an unbalanced force system. If a self-equilibrating system of forces were applied, there would be no acceleration and hence the total fictitious support must be identically zero.

An incidental advantage of this choice of support is that it automatically takes account of the loading and deformation due to self-weight of the ring, provided that appropriate values are used for the real support reactions.

\section{NOTATION AND SIGN CONVENTIONS}

The moment, $M$, and the force, $F$, transmitted across a typical cross-section of the ring can be conveniently represented in terms of their components along the co-ordinate directions $(r, \theta, z)$ as shown in Fig. 2.

The position of the section is defined by an angle, $\theta$, measured anticlockwise from a suitable datum when viewed from above in Fig. 2. The axial co-ordinate, $z$, is taken as positive upwards. Positive moments appear as clockwise when viewed along the arrows.

The deformation of the ring is defined by the displacement $u$ of the centroid of the ring section (components $u_{r}$ $\left.u_{\theta}, u_{2}\right)$ and by the angle of twist, $\beta$. Twist is regarded as positive if the ring cross-section is seen to rotate anticlockwise when viewed in the direction of increasing $\theta$.

\section{DERIVATION OF INFLUENCE FUNCTIONS}

The transverse and in-plane loading systems are not coupled and, hence, greater simplicity can be obtained by resolving the applied forces and moments into components along the axes. We therefore have to treat six elementary problems, each involving a single concentrated force (or moment) with an approximate fictitious support as defined in Section 2. The force can be applied at the section $\theta=0$ without loss of generality. The problem is to find the 


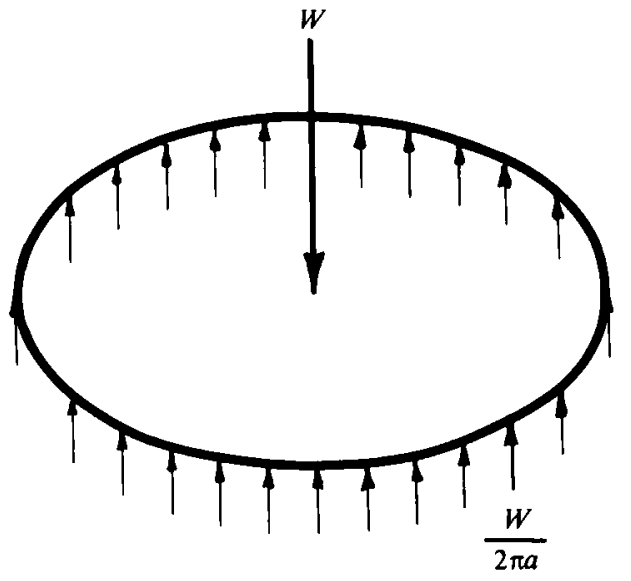

a

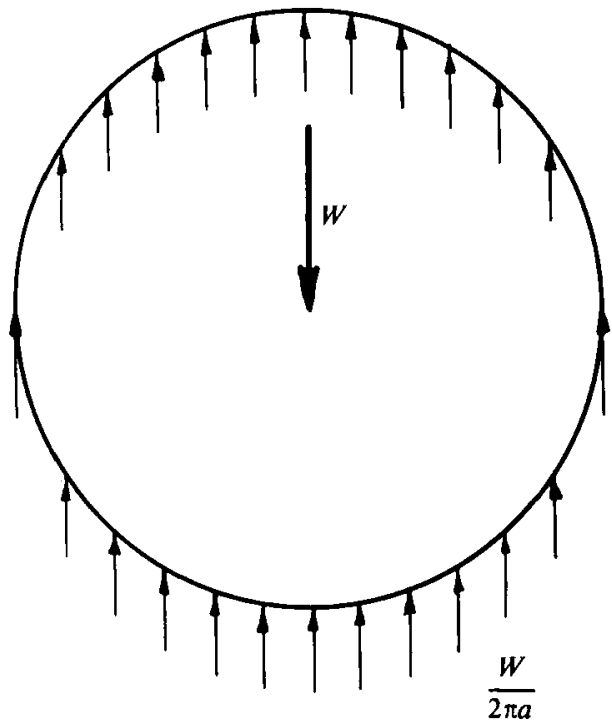

b
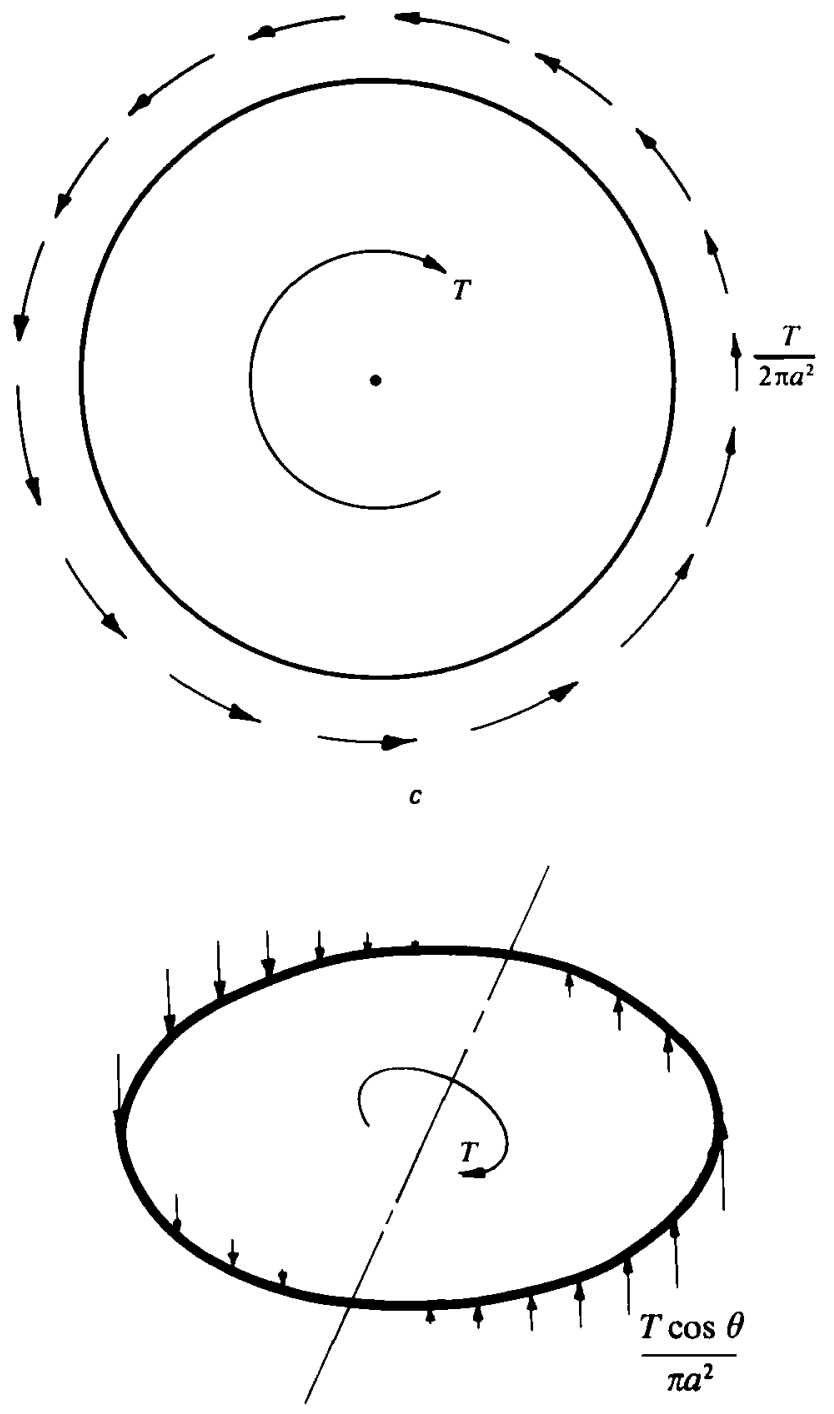

d

Fig. 1

transmitted force and moment at an arbitrary section and the deformed shape of the ring.

The solution is obtained by integrating the loaddeflection relations for the ring (see, for example, case 3) and applying appropriate symmetry or continuity conditions. The derivations (4) are elementary, though tedious, and only the results are given here in Appendix 1.

The expressions obtained for the displacements contain six arbitrary constants $\left(c_{1}-c_{6}\right)$ corresponding to the six

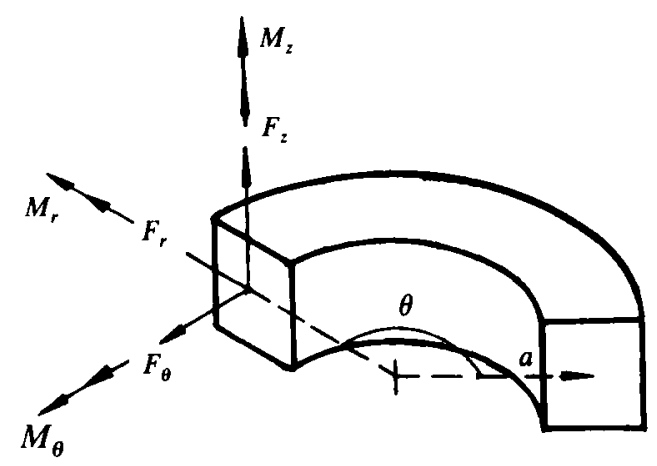

Fig. 2 degrees of freedom of rigid-body displacement of the ring. A pure rigid-body displacement is described by the equations

$$
\begin{aligned}
& \frac{1}{a}\left\{\frac{\mathrm{d} u_{r}}{\mathrm{~d} \theta}-u_{\theta}\right\}=-c_{3} \\
& u_{r}=c_{1} \cos \theta+c_{2} \sin \theta \\
& u_{\theta}=-c_{1} \sin \theta+c_{2} \cos \theta+a c_{3} \\
& \beta=c_{4} \cos \theta+c_{5} \sin \theta \\
& \frac{1}{a} \frac{\mathrm{d} u_{z}}{\mathrm{~d} \theta}=-c_{4} \sin \theta+c_{5} \cos \theta \\
& u_{z}=a c_{4} \cos \theta+a c_{5} \sin \theta+c_{6}
\end{aligned}
$$

These terms are omitted from the influence functions in the interests of brevity, but they must be reintroduced in the treatment of particular problems. The arbitrary constants are then determined from the boundary conditions of the problem. These boundary conditions may specify the slope of the ring as well as its displacement at a 
given point (as in a built-in support). Expressions for the in-plane slope

$$
\left\{\frac{1}{a} \frac{\mathrm{d} u_{r}}{\mathrm{~d} \theta}-\frac{u_{\theta}}{a}\right\}
$$

and the two transverse slopes

$$
\left(\beta \text { and } \frac{1}{a} \frac{\mathrm{d} u_{2}}{\mathrm{~d} \theta}\right)
$$

are therefore included in the list of influence functions given in Appendix 1.

The use of these results is illustrated in Section 5.

\section{APPLICATIONS}

If the loads on the ring are all known and the support system is statically determinate, the results given in Appendix 1 permit the solution to any problem to be written down as a sum, or as a definite integral, of the given functions.

For example, suppose that the ring in Fig. 3 is subjected

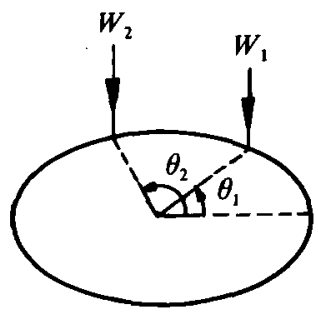

Fig. 3

to a self-equilibrating system of axial loads, $W_{i}$, acting at angular positions $\theta_{i}(i=1$ to $n)$, then the transverse bending moment, $M_{r}$, will be

$$
M_{r}=-\sum_{i=1}^{n} W_{i} a f_{5}\left(\theta-\theta_{i}\right)
$$

(See Table $1 b$, where $f_{5}(\theta)$ is given by equation (18).)

The results in Table 1 have been calculated for positive arguments in the range $(0 \leqslant \theta \leqslant 2 \pi)$. When $\left(\theta-\theta_{l}\right)$ is negative, $\left(2 \pi+\theta-\theta_{t}\right)$ should therefore be used in its place.

As a simple example of this procedure, we consider a uniform ring of weight $W_{0}$, radius $a$, supported in a vertical plane by a built-in support at one end of the horizontal diameter (see Fig. 4).

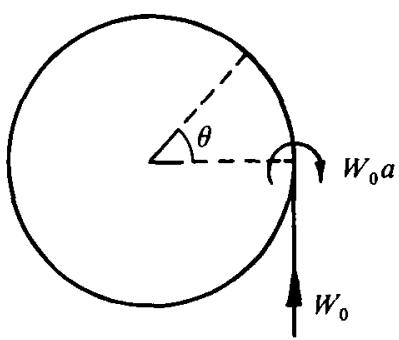

Fig. 4
The support introduces the following loading:

case 2: $W=-W_{0}$ at $\theta=0$

case 6: $T=+W_{0} a$ at $\theta=0$

The uniform self-weight of the ring need not be explicitly introduced, as it will be automatically invoked as a fictitious support for this otherwise unbalanced loading.

Using results for $M_{2}$ from Table $1 a$, we have

$$
\begin{aligned}
M_{z} & =-W_{0} a f_{6}(\theta-0)+W_{0} a\left\{f_{2}(\theta-0)+f_{6}(\theta-0)\right\} \\
& =W_{0} a\left\{\left(1-\frac{\theta}{\pi}\right) \frac{\cos \theta}{2}-\frac{\sin \theta}{4 \pi}\right\}
\end{aligned}
$$

from equations (18). The shear forces $F_{r}, F_{\theta}$ are readily found in the same way.

Applying the same procedure to the displacement equations, but introducing an arbitrary rigid-body displacement, we obtain

$$
\begin{aligned}
& u_{r}=-\frac{W_{0} a^{3}}{E I_{z}}\left\{f_{9}+f_{6}\right\}+c_{1} \cos \theta+c_{2} \sin \theta \\
& u_{\theta}=-\frac{W_{0} a^{3} f_{8}}{E I_{z}}-c_{1} \sin \theta+c_{2} \cos \theta+a c_{3} \\
& \frac{1}{a}\left\{\frac{\mathrm{d} u_{r}}{\mathrm{~d} \theta}-u_{\theta}\right\}=-\frac{W_{0} a^{2} f_{5}}{E I_{z}}-c_{3}
\end{aligned}
$$

The ring is built-in at $\theta=0$ and hence

$$
u_{r}=u_{\theta}=\frac{1}{a}\left\{\frac{\mathrm{d} u_{r}}{\mathrm{~d} \theta}-u_{\theta}\right\}=0 \text { at } \theta=0
$$

These three equations enable us to find the three constants:

$$
\left.\begin{array}{l}
c_{1}=0 \\
c_{2}=-\frac{3 W_{0} a^{3}}{4 \pi E I_{z}} \\
c_{3}=\frac{3 W_{0} a^{2}}{4 \pi E I_{z}}
\end{array}\right\}
$$

Substituting back into equations (9) and (10) gives

$$
\begin{aligned}
& u_{r}=-\frac{W_{0} a^{3}}{E I_{z}}\left(1-\frac{\theta}{2 \pi}\right) \frac{\theta \sin \theta}{4} \\
& u_{\theta}=-\frac{W_{0} a^{3}}{E I_{z}}\left\{\left(1-\frac{\theta}{2 \pi}\right) \frac{\theta \cos \theta}{4}\right. \\
& \left.-\left(1-\frac{\theta}{\pi}\right) \frac{\sin \theta}{4}-\frac{(1-\cos \theta)}{4 \pi}\right\}
\end{aligned}
$$

In particular, we note that the downward vertical displacement at $\theta=\pi / 2$ is

$$
-u_{r}\left(\frac{\pi}{2}\right)=\frac{3 W_{0} a^{3} \pi}{32 E I_{z}}
$$


Table 1

a In-plane loading

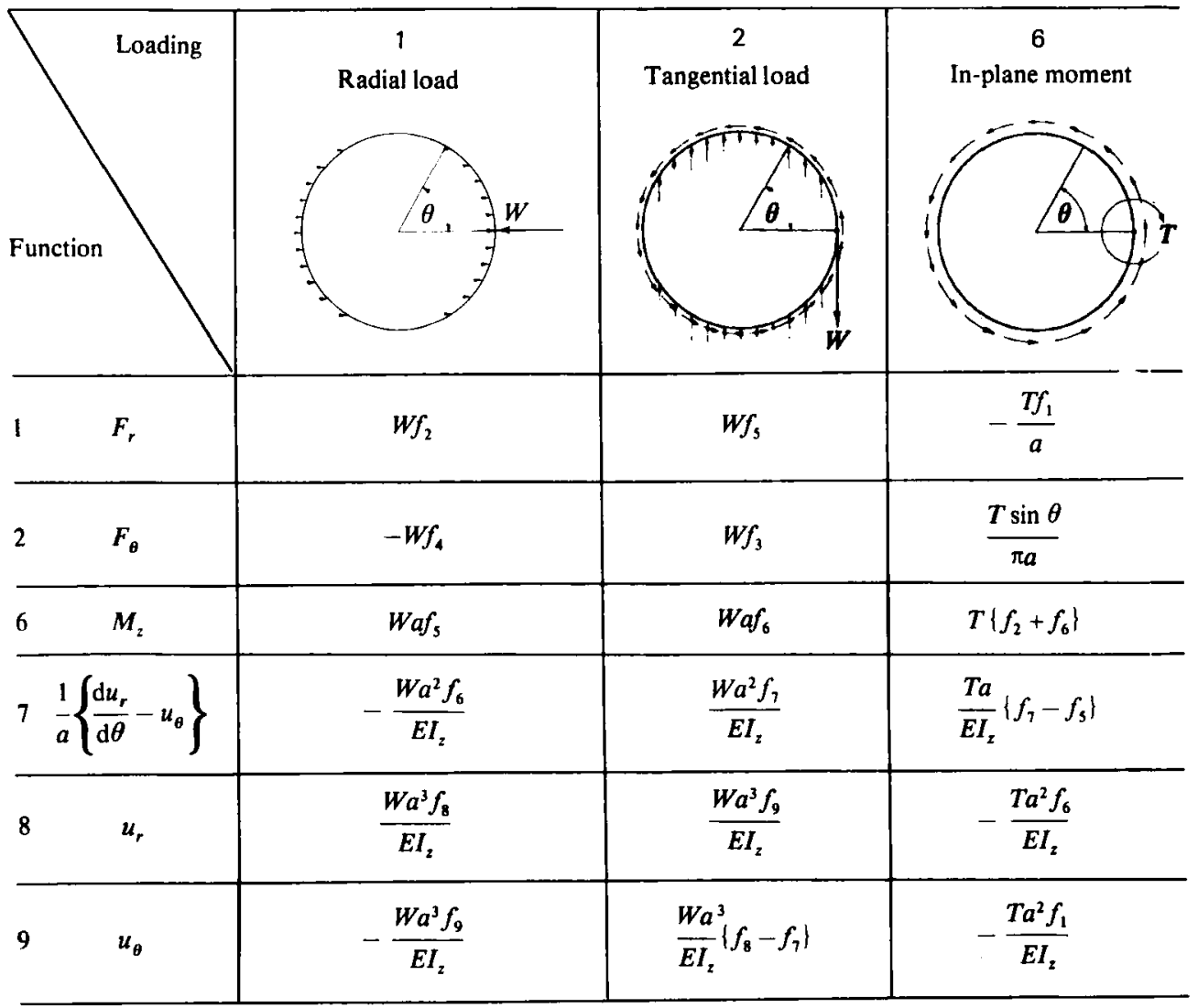

$b$ Transverse loading

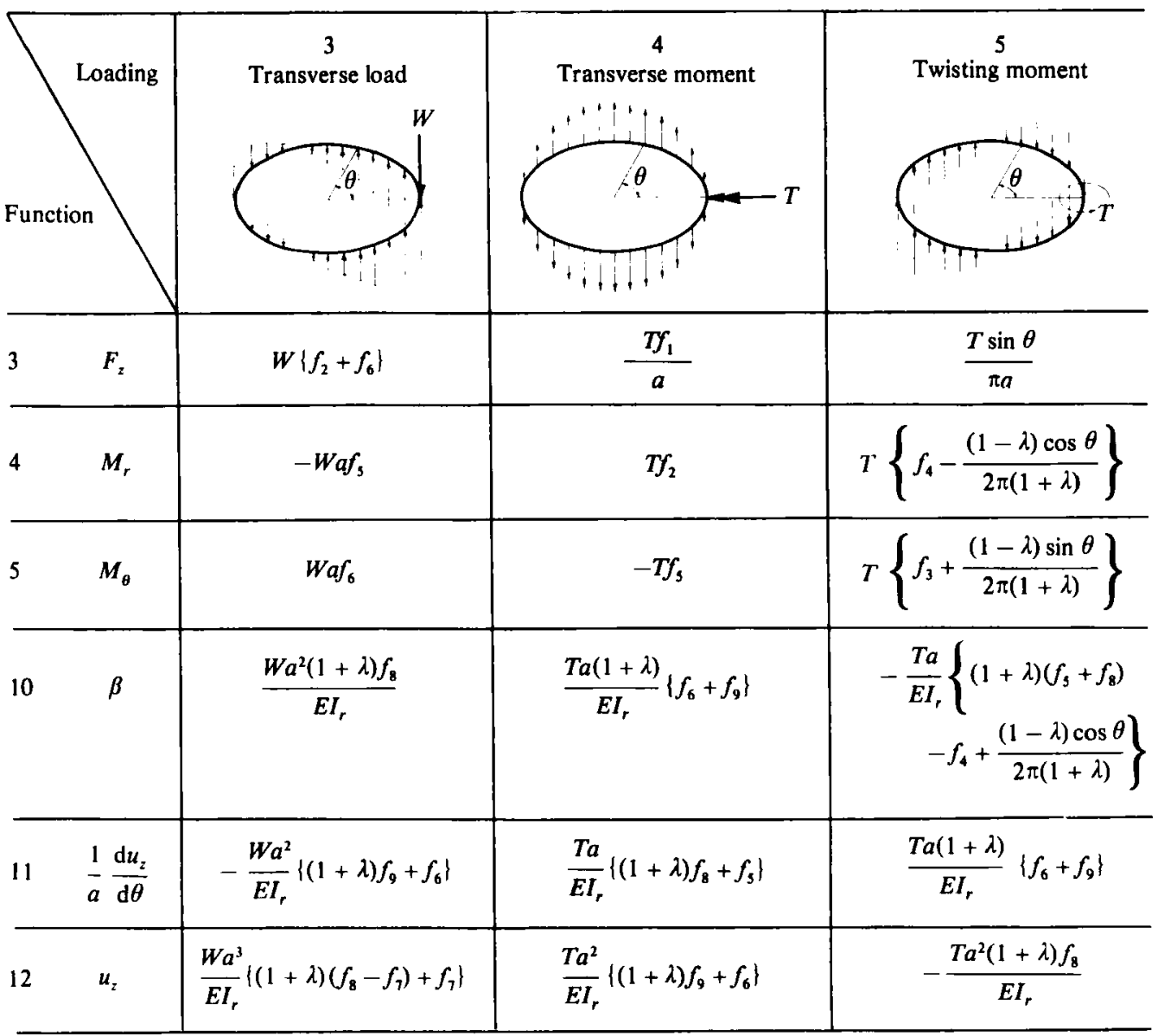


whereas at $\theta=\pi$ it is

$$
+u_{\theta}(\pi)=\frac{W_{0} a^{3}}{8 E I_{z}}\left(1+\frac{2}{\pi}\right)
$$

\section{CONCLUSIONS}

The solution to any problem for the complete, uniform, circular ring on a statically determinate support can be written down as a finite sum, or a definite integral, of the set of influence functions given in Appendix 1.

This degree of generality makes the method particularly suitable for incorporation into a computer-aided design routine, in which context it was originally developed.

\section{ACKNOWLEDGEMENTS}

The author is indebted to Davy Ashmore International Ltd, of Stockton, for permission to publish work which was carried out under their auspices as part of a computer-aided design programme.

\section{APPENDIX 1}

The influence functions can be expressed concisely in terms of the nine functions defined below.

$$
\left.\begin{array}{l}
f_{1}=\frac{\cos \theta}{\pi}+\frac{1}{2 \pi} \\
f_{2}=\left(1-\frac{\theta}{\pi}\right) \frac{\cos \theta}{2}-\frac{\sin \theta}{4 \pi} \\
f_{3}=\left(1-\frac{\theta}{\pi}\right) \frac{\cos \theta}{2}+\frac{\sin \theta}{4 \pi} \\
f_{4}=\left(1-\frac{\theta}{\pi}\right) \frac{\sin \theta}{2}+\frac{\cos \theta}{4 \pi} \\
f_{5}=\left(1-\frac{\theta}{\pi}\right) \frac{\sin \theta}{2}-\frac{\cos \theta}{4 \pi}-\frac{1}{2 \pi}
\end{array}\right\}
$$

$$
\left.\begin{array}{l}
f_{6}=\left(1-\frac{\theta}{\pi}\right) \frac{(1-\cos \theta)}{2}-\frac{3 \sin \theta}{4 \pi} \\
f_{7}=\left(1-\frac{\theta}{\pi}\right) \frac{\sin \theta}{2}-\frac{5 \cos \theta}{4 \pi}+\frac{1}{2 \pi} \\
-\frac{\theta}{2}\left(1-\frac{\theta}{2 \pi}\right) \\
f_{8}=\left(1-\frac{\theta}{2 \pi}\right) \frac{\theta \cos \theta}{4}-\left(1-\frac{\theta}{\pi}\right) \\
\times \frac{\sin \theta}{4}+\frac{(1-\cos \theta)}{2 \pi} \\
f_{9}=\left(1-\frac{\theta}{2 \pi}\right) \frac{\theta \sin \theta}{4}-\left(1-\frac{\theta}{\pi}\right) \frac{(1-\cos \theta)}{2}
\end{array}\right\} \begin{gathered}
(18 \\
\operatorname{cont} .)
\end{gathered}
$$

In the equations that follow, $I_{r}, I_{z}$ are the second moments of area of the ring section about centroidal axes parallel to the $r, z$ directions respectively, $E$ is Young's modulus, and $G K$ is the torsional rigidity of the section. The ratio $\lambda$ defined by

$$
\lambda=\frac{E I_{r}}{G K}
$$

occurs in the results for transverse bending of the ring.

Using this notation, the influence functions take the forms shown in Table 1 .

\section{APPENDIX 2}

\section{REFERENCES}

(1) ROARK, J. R. Formulas for stress and strain 1954 (McGrawHill, New York)

(2) BLAKE, A. 'How to find deflection and moment of rings and arcuate beams', Product Engng 1963, 70-81.

(3) CASE, J. The strength of materials 1938, 3rd edn, 389 (Edward Arnold, London).

(4) BARBER, J. R. The use of influence functions to find the forces and displacements in a complete circular ring Internal Report Ta.37, 1976 (University of Newcastle Upon Tyne). 